\title{
Fibrotic Lung Toxicity Induced by Hydroxycarbamide
}

\author{
ELENA BARGAGLI ${ }^{1}$, MARCO PALAZZI $^{1}$, FRANCESCO PERRI $^{1}{ }$, ELENA TORRICELLI ${ }^{1}$, \\ ELISABETTA ROSI ${ }^{1}$, ALESSANDRA BINDI ${ }^{2}$, MASSIMO PISTOLESI ${ }^{1}$ and LUCA VOLTOLINI ${ }^{3}$ \\ ${ }^{1}$ Section of Respiratory Medicine, Department of Clinical and Experimental Biomedical Sciences, \\ ${ }^{2}$ Section of Radiology, and ${ }^{3}$ Thoracic Surgery Unit, Careggi University Hospital, Florence, Italy
}

\begin{abstract}
A patient treated for 4 months with hydroxycarbamide (hydroxyurea) for chronic myelomonocytic leukemia was admitted to hospital for recently developed severe dyspnea and acute respiratory failure. The computed tomographic scan of the chest showed diffuse ground glass opacities, some centrilobular low-density nodules (resembling hypersensitivity pneumonitis-like pattern), and minimal interstitial reticulation of the subpleural region. The analysis of bronchoalveolar lavage fluid excluded infection, as did serological examinations. The patient was started on oxygen therapy and with relief of thrombocytopenia and suspected hemolytic anemia, hydroxyurea treatment was discontinued. The patient underwent steroid therapy, with a rapid progressive improvement of clinical and radiological features. As hydroxyurea is increasingly used for a number of systemic disorders, physicians must be aware of its potential lung toxicity, requiring immediate cessation of the treatment and empiric corticosteroid therapy.
\end{abstract}

Hydroxycarbamide (hydroxyurea) is a drug widely indicated for the treatment of myeloproliferative disorders (chronic myeloid leukemia) and sickle cell anemia (1-3). As a rule, this antineoplastic drug is well tolerated (1), and is commonly used for ovarian neoplasms, squamous cancer of the head and neck, and glioblastoma multiforme. The most common toxic effects include hematological alterations associated with bone marrow failure and T-cell reduction, thrombocytopenia, anemia and neutropenia (1-3). Other common side-effects (10\% or more) include asthenia,

This article is freely accessible online.

Correspondence to: Elena Bargagli, MD, Ph.D., Department of Clinical and Experimental Biomedical Sciences, University of Florence, Florence, Italy. Mobile: +39 3391590310, e-mail: bargagli2@gmail.com

Key Words: Hydroxycarbamide, hydroxyurea, lung toxicity, interstitial pneumonitis, case report. azoospermia, malaise, dysuria, blood creatinine increase, disorientation, hallucination, fever, gastrointestinal disorders (such as pancreatitis, nausea, vomiting, diarrhea, stomatitis, mucositis, dyspepsia, melena), and dermatological lesions (vasculitis, alopecia, popular rush, skin atrophy and exfoliation or ulcers) (1-3). In 1-10\% of cases, the drug can lead to hepatotoxicity, hepatitis and neuropathy. Potential carcinogenic effects include an increased risk of skin cancer. Hydroxycarbamide can induce lung toxicity with pulmonary edema, lung infiltration and dyspnea (4-8), and pulmonary fibrosis in a minority of patients (5). Herein we report the clinical history of a patient experiencing acute interstitial lung injury secondary to hydroxyurea administration.

\section{Case Report}

An 83-year-old man was admitted to our hospital for acute respiratory distress. The patient was a former smoker with only 5 pack/years of exposition. The patient's past clinical history revealed systemic arterial hypertension, treated with calcium channel blockers. Twenty years earlier he had undergone surgery for an abdominal liposarcoma and during the previous 4 months had started hydroxyurea treatment (500 mg/day), following the diagnosis of chronic idiopathic myelofibrosis. The patient complained of breathlessness and a dry cough, which had worsened over the previous week. He was afebrile, the arterial blood gas analysis revealed severe hypoxemia and routine blood tests showed thrombocytopenia $\left(63,000 \times 10^{6} / 1\right)$. The electrocardiogram was normal and echocardiography excluded acute heart failure. The serological microbiological assays revealed an uncertain positivity for Mycoplasma pneumonia, while blood cultures were negative. Chest examination revealed bilateral basal crackles and the chest X-ray showed bilateral diffuse shadows. The computed tomographic (CT) scan of the chest excluded pulmonary embolism and showed ground glass opacities with mosaicism in both lungs. In the upper lobes, small ground-glass zones were observed. Some centrilobular low density nodules resembling a hypersensitivity pneumonitis-like pattern and minimal interstitial reticulation 

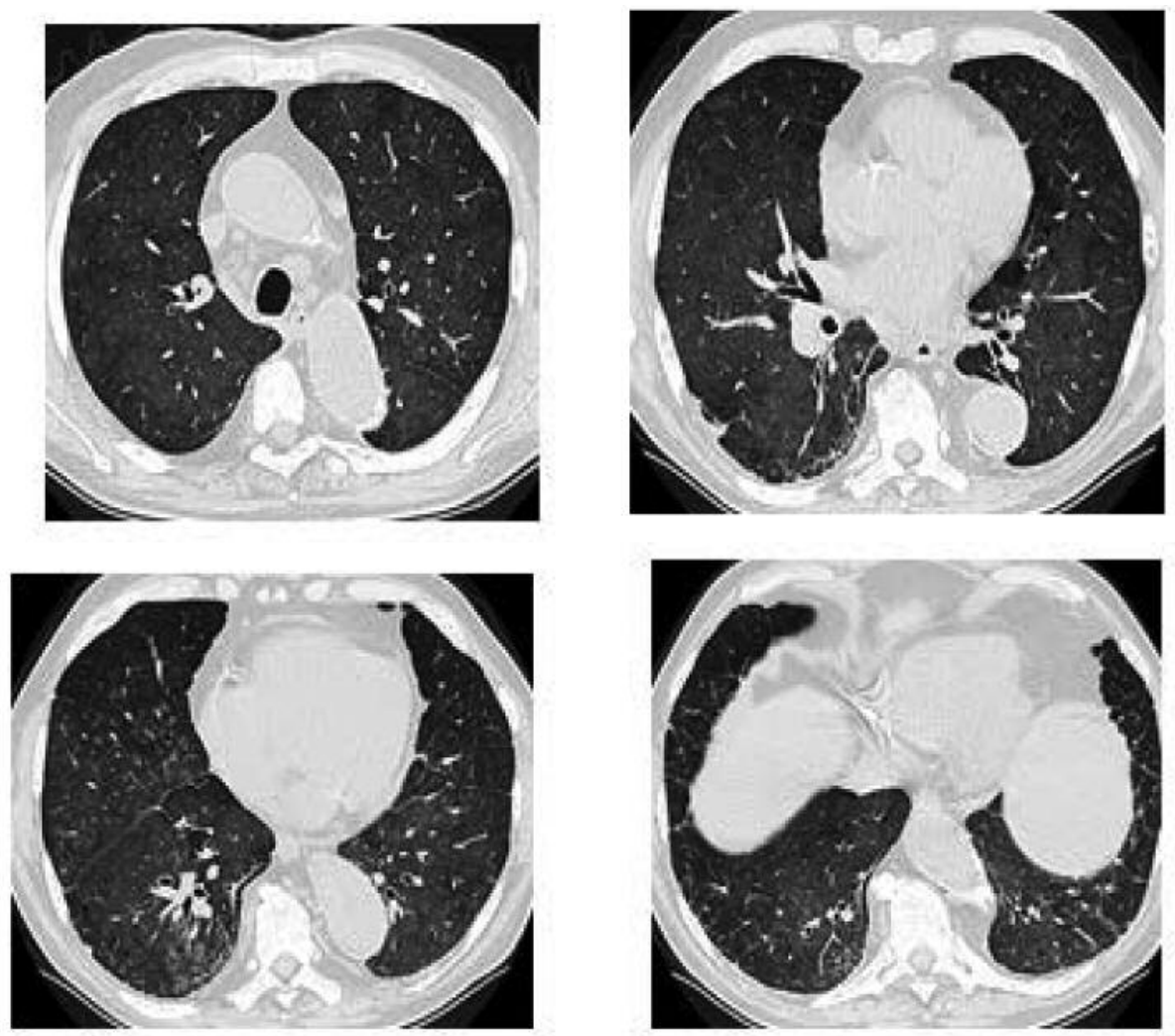

Figure 1. High-resolution computed tomography of the chest showing diffuse ground-glass opacities (a), bilateral peripheral reticulations (b), areas of subpleural thickening (c), and bilateral centrilobular low-density nodules $(d)$.

of the subpleural regions were observed, especially in the dorsal parts of lower lobes. Some mediastinal and hilar lymphadenopathies were also present without pleural or pericardial effusions (Figure 1). The patient was started on oxygen therapy and intravenous broad-spectrum antibiotics. Bonchoscopy with bronchoalveolar lavage was performed. Microscopy and cultures for mycobacteria, common bacteria, viruses and fungi were negative. Polymerase chain reaction for Legionella pneumophila, Chlamydia pneumoniae and Mycoplasma pneumoniae, Herpes simplex virus (HSV) 1 and 2, Adenovirus and Pneumocystis jiroveci were also negative, as well as galactomannan and aspergillus antigen tests. The cytological examination and transbronchial needle aspiration of the right hilar lymph nodes (station $11 \mathrm{R}$ ) excluded malignant abnormalities. The cellular count in bronchoalveolar lavage revealed $40 \%$ macrophages, $55 \%$ lymphocytes (with a normal CD4/CD8 ratio), and 5\% neutrophils.

With the relief of thrombocytopenia and suspected hemolytic anemia, in accordance with hematologists, hydroxyurea treatment was discontinued. A diagnosis of interstitial pneumonia associated with acute respiratory failure was made and we treated the patient with oxygen, low dose of diuretics, oral azithromycin and low dose of oral steroids (16 mg/day prednisolone). A month later the patient was referred to the pulmonologist for examination. Lung function tests evidenced a mild obstructive syndrome with a moderateto-severe reduction of diffusion capacity for carbon monoxide. The results of arterial blood gas analysis with $2 \mathrm{1} / \mathrm{min}$ of $\mathrm{O}_{2}$ were: $\mathrm{paO}_{2}=69.9 \mathrm{mmHg}, \mathrm{paCO}_{2}=28.9 \mathrm{mmHg}$, and $\mathrm{pH}$ of 7.45 . After 4 weeks, the abnormal chest radiography and CT findings had improved, therefore it was decided to increase the amount of prednisolone to $50 \mathrm{mg} /$ day. Further improvements of the pulmonary situation were recorded after 3 months and adequate oxygenation became possible with $1.5 \mathrm{l} / \mathrm{min}$ of $\mathrm{O}_{2}$. The hydroxyurea treatment was not reintroduced and the patient is stable, his respiratory symptoms are improved as well as the lung function test parameters and the blood gas analysis. He requires oxygen only under effort.

\section{Discussion}

This case report underlines the risk of lung fibrotic toxicity in patients treated with hydroxyurea for a myeloproliferative 
disease. This is a rare event and very few cases have been reported in the literature (5-12). In this limited population, the interstitial lung toxicity generally occurred after a few weeks of exposure to hydroxyurea (9), while in our patient after 15 weeks. Lung toxicity is usually associated with dyspnea and fever (5-7). Fever may be the only sign of a drug reaction, resolving with withdrawal and recurring at rechallenge (4). As previously found by Cleverley et al. (13) in a similar case, fever was absent in our patient while dyspnea was severe, the lung toxicity was mainly documented by $\mathrm{CT}$ scan alterations and discontinuing hydroxyurea resulted in the improvement of clinical and radiological conditions. However, hydroxyurea-induced pneumonia was reported as being lethal in a recent case report in which steroids were also used at high dose with no benefit; the disease diagnosis was confirmed after autopsy and by a drug lymphocyte stimulation test positive for hydroxyurea (11).

As suggested by Rossi et al. (8) and others (11-13), the identification of ground-glass opacities at high resolution CT scan of the chest in a patient with acute respiratory symptoms treated with hydroxyurea requires a complex differential diagnosis with bacterial or viral pneumonitis, respiratory infections (aggravated by myelosuppressive therapy), and pulmonary hypertension. In our patient, the microbiological examinations allowed for exclusion of infections and the radiological high-resolution CT features were consistent with the phenomenon of drug-induced lung toxicity being similar to an hypersensitivity pneumonitis-like pattern and comparable to a histological picture of diffuse alveolar damage, already reported in Pneumotox associated with hydroxyurea exposure (14).

Mechanisms of hydroxyurea toxicity are unclear and further research is needed (15-17). This drug is increasingly used for a number of malignant and non-malignant disorders, however, as underlined by this case report, it can induce severe interstitial pneumonitis with diffuse alveolar damage and radiological pulmonary infiltrates requiring an early cessation of the treatment and empiric corticosteroid therapy (17). It is necessary that hematologists and physicians are aware of the lung toxicity of hydroxyurea and its severe and potentially life-threatening effects.

\section{Conflicts of Interest}

All the Authors declare that they have no conflict of interest related with this topic of research.

\section{References}

1 Nevitt SJ, Jones AP and Howard J: Hydroxyurea (hydroxycarbamide) for sickle cell disease. Cochrane Database Syst Rev 4: CD002202, 2017.
2 Finazzi G, Ruggeri M, Rodeghiero F and Barbui T: Hydroxyurea for patients with essential thrombocythemia and a high risk of thrombosis. N Engl J Med 332: 1132-1136, 1995.

3 Grandi V, Delfino C and Pimpinelli N: Ingenol mebutate in the treatment of 'hydroxyurea-induced squamous dysplasia': a single-centre experience. J Eur Acad Dermatol Venereol 30(7): 1129-1132, 2016.

4 Blanc PL, Joasson JM, Hammoud $\mathrm{H}$ and Matussière Y: Acute interstitial pneumopathy with fever caused by hydroxyurea. Presse Med 29(5): 242-245, 2000.

5 Internullo M, Giannelli V, Sardo L, Antonaglia C, Villani T, Angelici $\mathrm{E}$ and Palange P: Hydroxyurea-induced interstitial pneumonitis: case report and review of the literature. Eur Rev Med Pharmacol Sci 18(2): 190-193, 2014.

6 Kavuru MS, Gadsden T, Lichtin A and Gephardt G: Hydroxyurea-induced acute interstitial lung disease. South Med J 87(7): 767-769, 1994.

7 Sandhu HS, Barnes PJ and Hernandez P: Hydroxyurea-induced hypersensitivity pneumonitis: a case report and literature review. Can Respir J 7: 491-495, 2000.

8 Rossi SE, Erasmus JJ, McAdams HP, Sporn TA and Goodman PC: Pulmonary drug toxicity: radiologic and pathologic manifestations. Radiographics 20: 1245-1259, 2000.

9 Hennemann B, Bross KJ, Reichle A and Andreesen R: Acute alveolitis induced by hydroxyurea in a patient with myeloproliferative syndrome. Ann Hematol 67: 133-134, 1993.

10 Loo PS, Khan M, Currie GP, Husain E and Kerr KM: Hydroxycarbamide-induced pneumonitis. Histopathology 55: 234-236, 2009.

11 Imai H, Matsumura N, Yamazaki Y, Kanayama Y, Masuda T, Kuwako T, Kamide Y, Tomizawa T, Matsumoto S, Mitsui T, Kaira K, Ono A, Koga Y, Shibusawa N, Sunaga N, Hisada T, Yokoo $\mathrm{H}$ and Yamada M: Hydroxyurea-induced pneumonitis in a patient with chronic myelomonocytic leukemia: An autopsy case. Intern Med 54(24): 3171-3176, 2015.

12 Quintas-Cardama A, Pérez-Encinas M, Gonzalez S, Bendaña A and Bello JL: Hydroxyurea-induced acute interstitial pneumonitis in a patient with essential thrombocythemia. Ann Hematol 78: 187-188, 1999.

13 Cleverley JR, Screaton NJ, Hiorns MP, Flint JD and Müller NL: Drug-induced lung disease: high-resolution CT and histological findings. Clin Radiol 57: 292-299, 2002.

14 Bonniaud P, Georges M, Favrolt N and Camus P: Drug induced ILD. Rev Prat 64(7): 951-956, 2014.

15 Bajwa AA, Usman F, Wolfson D, Laos LF and Cury JD: A 62year-old woman with dyspnea, leukocytosis, and diffuse groundglass opacities. Chest 137(6): 1470-1473, 2010.

16 Rumi E, Passamonti F, Boveri E, De Amici M, Astori C, Braschi M, Castagnola C, Magrini U, Cazzola $M$ and Lazzarino $M$ : Dyspnea secondary to pulmonary hematopoiesis as presenting symptom of myelofibrosis with myeloid metaplasia. Am J Hematol 81(2): 124-127, 2006.

17 Girard A, Ricordel C, Poullot E, Claeyssen V, Decaux O, Desrues B, Delaval P and Jouneau S: Hydroxyurea-induced pneumonia. Rev Mal Respir 31(5): 430-434, 2014.

Received August 16, 2017

Revised September 8, 2017

Accepted September 14, 2017 